\title{
Severe new limits on the host galaxies of Gamma-Ray Bursts
}

\author{
B.E. Schaefer \\ Department of Physics, Yale University, New Haven, CT 06520-8121, U.S.A.
}

Received December 29, 1998; accepted June 9, 1999

\begin{abstract}
Most burst models require bursters to reside in normal host galaxies with peak luminosities of $L=$ $110^{57}$ photon $\mathrm{s}^{-1}$ or $L=210^{58}$ photon $\mathrm{s}^{-1}$. These models can be directly tested by looking for host galaxies in small GRB error boxes in either of two data sets: (1) 8 optical transients detected for SAX bursts and (2) 23 of the alltime bright bursts. If the hosts are normal galaxies, then

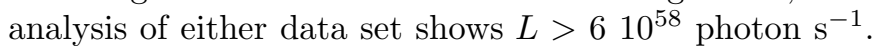
This result strongly rejects both luminosity cases provided bursters appear in normal host galaxies, with the bright burst sample being free of problems relating to galaxy evolution. In conclusion, bursters are either more distant than allowed for in the star-formation-rate scenario or they are not in normal galaxies.
\end{abstract}

Key words: gamma-rays: bursts

\section{Introduction}

What is the average peak luminosity of Gamma-Ray Bursts (GRBs)? We now know that they are indeed cosmological in distance, yet there is still a wide range of possible luminosities. If the number density does not evolve, then fits to the $\log N-\log P$ curve suggest that the peak luminosity is $110^{57}$ photon $\mathrm{s}^{-1}$ or roughly $610^{50} \mathrm{erg} \mathrm{s}^{-1}$. (Peak luminosities in photon units are all for $50-300 \mathrm{keV}$ with $256 \mathrm{~ms}$ time bins, while those in ergs are for $30-2000 \mathrm{keV}$.) If the number density traces the star formation rate (SFR) of our Universe, then fits to the $\log N-\log P$ curve suggest that the peak luminosity is $210^{58}$ photon $\mathrm{s}^{-1}$ or roughly $110^{52} \mathrm{erg} \mathrm{s}^{-1}$. At this meeting, there were several suggestions that the peak luminosity might be substantially larger than even the SFR value.

The average luminosity of GRBs still has large uncertainties. The GRB optical transients (OTs) with a reported red shift yield luminosity values which range over roughly five orders of magnitude. GRB 971214 has a galaxy with red shift $z=3.42$ and a luminosity of $110^{59}$ photon $\mathrm{s}^{-1}$. GRB 980703 has an optical transient with a red shift of $z=0.966$ which corresponds to a luminosity of $110^{58}$ photon s ${ }^{-1}$. GRB 970508 has an optical transient with a red shift of $z=0.83$ and a luminosity of $210^{57}$ photon s $^{-1}$. GRB 970425 has an associated unusual supernova at a red shift of $z=0.0085$ for a luminosity of $110^{54}$ photon $\mathrm{s}^{-1}$. Although the optical red shift measures provides promise of definitive answers within a few years, the current situation has no clear conclusion.

In the mean time, other measured quantities give information on the distance and luminosity distribution of bursters. In particular, the limits on apparent magnitudes of possible host galaxies inside the small GRB optical transient boxes carries information on the GRB distance scale. The idea is that nearby (low luminosity) events should have their hosts appear relatively bright while distant (high luminosity) events should have their hosts appear systematically faint. This paper gives the result from an analysis on the limits to possible host galaxies inside the error boxes for eight GRBs with optical transients.

\section{GRB/OT boxes}

The particular analysis method is that originally proposed by Fenimore et al. (1993) as elaborated by Schaefer (1999). In this method, for some assumed peak luminosity, the limit on the apparent magnitude of the host is translated into a fraction $\left(F_{\min }\right)$ of the faint end of the Schechter luminosity function in which the host must lie. If bursts reside in normal host galaxies, then the average $\left\langle F_{\min }\right\rangle$ value should be near a half. With foreground unrelated galaxies and with nondetection thresholds, $\left\langle F_{\min }\right\rangle$ can only be larger than half. If bursters do not reside in normal hosts, then the $\left\langle F_{\min }\right\rangle$ may be smaller than a half. The analysis procedure is to test a range of assumed peak luminosities to find the values consistent with the presence of normal host galaxies. For the events with measured red shifts, the implied distances have been adopted, except for 
Table 1. Host galaxy Data: GRB/OT boxes

\begin{tabular}{|c|c|c|}
\hline GRB & $\begin{array}{c}P_{256} \\
\left(\mathrm{ph} \mathrm{s}^{-1} \mathrm{~cm}^{-2}\right)\end{array}$ & $\begin{array}{l}V_{\text {host }} \\
(\text { mag })\end{array}$ \\
\hline 970228 & 10 & $\geq 24.88$ \\
\hline 970508 & 1.2 & $\geq 25.55$ \\
\hline 971214 & 2.3 & $\geq 25.56$ \\
\hline 980326 & 1.2 & $\geq 25.00$ \\
\hline 980329 & 13.3 & $\geq 25.40$ \\
\hline 980519 & 4.7 & $\geq 25.14$ \\
\hline 980613 & 0.6 & $\geq 24.42$ \\
\hline 980703 & 2.6 & $\geq 22.39$ \\
\hline
\end{tabular}

the GRB 970425 event which is ignored as it must be a special case.

The peak fluxes and limits on the host $V$ magnitudes (corrected for extinction by our Milky Way) have been collected for eight GRBs with small OT boxes (see Table 1). The analysis shows that $\left\langle F_{\min }\right\rangle=0.19 \pm 0.07$ for an assumed peak luminosity as given for the no-evolution case. Only when the assumed peak luminosity rises as large as that for the SFR case does $\left\langle F_{\min }>\right.$ rise to within onesigma of a half. For $\left\langle F_{\min }>\right.$ to be $\geq 0.5$, the average peak luminosity must be $\geq 610^{58}$ photon $\mathrm{s}^{-1}$. The effects of a broad luminosity function on the model $\left\langle F_{\min }\right\rangle$ value will be to form an appropriately weighted average over the monoenergetic $F_{\min }$ values, so that the observed $\left.<F_{\min }\right\rangle$ should be compared with the model value for the average peak luminosity. Thus, the result for the eight GRB/OT boxes is that the no-evolution distance scale is rejected, the SFR distance scale is close to being rejected, while the preferred peak luminosity is $\geq 610^{58}$ photons $\mathrm{s}^{-1}$ (provided bursters reside in normal galaxies).

This conclusion has some definite weaknesses relating to the lack of knowledge of galaxies at high red shift. In particular, at red shifts of $z>\sim 1$, the normal galaxy $K$ corrections are nearly unknown, the luminosity function could be substantially different from the Schechter function, there could be significant unaccounted absorption from dust at early epoch, and even uncertainties in the cosmological parameters start to matter. Given these large potential problems, we should conclude that the SFR model is not rejected. However, the no-evolution model places the bursts at low enough of a red shift that the uncertainties are unlikely to change the conclusion that this distance scale is rejected.

\section{Small IPN boxes}

This conclusion is actually an old one (e.g. Schaefer 1992; Schaefer et al. 1997) and can be substantially improved upon. The improvement comes with using the smallest error boxes produced by the Interplanetary Network (IPN), since these bursts are greatly brighter than those with OT boxes. For the 16 IPN boxes with a size less than 25 square arc-minutes and more than $10^{\circ}$ from the galactic equator, the maximum and median and minimum $P_{256}$ values are 73 and 44 and 15 photon $\mathrm{s}^{-1} \mathrm{~cm}^{-2}$. In contrast, the eight GRBs with OT boxes have maximum and median and minimum $P_{256}$ values of 13.3 and 2.4 and 0.6 photon $\mathrm{s}^{-1} \mathrm{~cm}^{-2}$. Thus, the IPN bursts are $\sim 16$ times brighter than the OT bursts. Approximately, the IPN bursts will be four times closer. This is important since these all-time bright bursts will be sufficiently close such that all of the problems that plague the faint and distant OT bursts will be small. From detailed calculations for the SFR case, the IPN bursts have median red shifts of 0.6 while the OT bursts have median red shifts of 2.2. Thus, the bright IPN bursts can be used with minimal uncertainties related to $K$ corrections, luminosity functions, anomalous dust extinction, and cosmology.

Schaefer (1999) analyses the limits on host galaxy brightnesses from the compilation of Schaefer et al. (1998) with the same method as reported in this paper. The result is that $\left\langle F_{\min }\right\rangle=0.14 \pm 0.05$ for the no-evolution case, $\left\langle F_{\min }>=0.29 \pm 0.12\right.$ for the SFR case, the minimum peak luminosity acceptable at the one-sigma level is $610^{58}$ photon $\mathrm{s}^{-1}$, while the best estimate of $\left\langle F_{\min }\right\rangle$ is

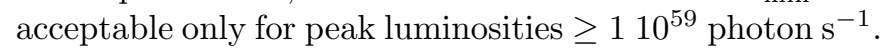
These limits are more restrictive than those from the OT bursts, and the uncertainties associated with high red shift are minimal.

From either the IPN or OT bursts, I conclude that the average peak luminosity of GRBs must be $\geq 610^{58}$ photon $\mathrm{s}^{-1}$ if the bursters reside in normal galaxies. This corresponds to the faint BATSE bursts typically at red shifts of 5.9. Alternatively, GRBs might reside in systematically and greatly subluminous galaxies or perhaps even outside galaxies.

\section{References}

Fenimore E.E., et al., 1993, Nat 366, 40

Schaefer B.E., 1992, in Gamma Ray Bursts, Ho C., Epstein R. $\&$ Fenimore E. (eds.). Cambridge: Cambridge Univ. Press, p. 107

Schaefer B.E., 1999, ApJL (in press)

Schaefer B.E., et al., 1997, ApJL 489, 693

Schaefer B.E., et al., 1998, ApJS 118, 353 